\title{
Fuzzy-Ant Colony based Routing on Road Networks
}

\author{
S. Saravanan ${ }^{1}$, A. Jayanthiladevi ${ }^{2 *}$, M. Geetha ${ }^{3}$ \\ ${ }^{1}$ Department of Computer Science and Engineering, Dhirajlal Gandhi College of Technology, Salem, India \\ ${ }^{2}$ Department of Master of Computer Applications, Jain University, Bangalore, India \\ ${ }^{3}$ Department of Master of Computer Applications, Sona College of Technology, Salem, India \\ *Corresponding author, e-mail: jayanthilamca@gmail.com
}

\begin{abstract}
Route selection is essential in everyday life. We have several algorithms for detecting efficient route on Large Road Networks. This paper introduces the hierarchical community, is presented. It splits large road networks into hierarchical structure. It introduces a multi parameter route selection system which employs Fuzzy Logic (FL) and ant's behavior in nature is applied to the dynamic routing. The important rates of parameters such as path length and traffic are adjustable by the user. The purposes of the new hierarchical routing algorithm significantly reduce the search space. We develop a community-based hierarchical graph model that supports Dynamic, efficient route computation on large road networks.
\end{abstract}

Keywords: Hierarchical community, traffic routing, dynamic parameters, fuzzy logic, Ant- colony algorithm

Copyright (C) 2016 APTIKOM - All rights reserved.

\section{Introduction}

We propose a routing strategy to improve the transportation efficiency on complex networks. Instead of using the routing strategy for shortest paths. In route selection problem, typically a pair of origin and destination is given, while there are many possible ways for selection. The objective is to find a desired destination through a faster way, the cost calculated for different possible directions. The most classical shortest path algorithm is the Dijkstra algorithm [1], whose complexity is $\mathrm{O}(|\mathrm{E}|+|\mathrm{V}| \log |\mathrm{V}|)$, where $|\mathrm{V}|$ is the number of vertices and $|\mathrm{E}|$ is the number of arcs. The Dijkstra algorithm performs well on a relatively small network.

The proposed approach combines both static and dynamic. Static means it splits large rod network into a hierarchical structure. Dynamic means the rates of parameter such as path length and traffic are adjustable by the user.

The proposed system roughly divided into two Components.

\subsection{Constructing the Hierarchical Community}

One component makes use of the intrinsic hierarchical topologies of road networks [2]-[7], which casts a road network into a hierarchical representation according to road categories, road lengths, speed limits, number of lanes, etc. Taking road category criterion as an example, Liu [3] suggested that major roads and express-ways themselves form a higher level network, which is connected and much more sparse, and this higher level network partitions the whole network into several sub networks. In this approach, each hierarchy forms a connected network; the higher the hierarchy, the sparser the network would be. This implies that adjacent sub networks are somewhat overlapping, and they share some important vertices and arcs, which happen to be its higher level network. The routing process will be accelerated since the search will go ascending to its higher level of hierarchy via these important vertices (named entry or exit points in the literature) and descending when the destination is approaching. It focuses more on how to partition and manage a network such that the number of boundary/ border nodes for each sub network is uniform and minimized, the sub networks are approximately of equal size, and so on (to reduce preprocessing cost) [9]-[14]. In this approach, each sub network forms an isolated part, and different parts are connected together via intergroup arcs. A search on the original network will switch to a higher level network, and time efficiency is improved. 


\subsection{Dynamic Route Selection}

The second component is a dynamic route selection. Fuzzy logic is a powerful tool for optimizing route selection from multi travel path. Ant Colony Optimization (ACO) approach solve spatial reconfiguration problem of multi model travel network. An optimum route refers to a route that attempts to satisfy all desired parameters of a user. These parameters are "Distance," "Traffic," "Tollgate", "Incident Risk", parameters. The combination of FL with ACO recognizes best direction with higher reliability. Wireless Sensor Network (WSN) to predict dynamically changed traffic data.

The rest of the paper is organized as follows: Section II describes the Hierarchical Community based graph model. Section III introduces the proposed route selection system. Section IV gives the simulation results. Section V concludes the paper.

\section{Hierarchical Community Based Graph Model}

Communities are groups of vertices. Networks often show a hierarchical structure of Communities nested within each other. These communities used to give better visualizing the structure of networks [8].

In a road network where streets are mapped as edges and intersections as vertices, the intersections that locate close in a small region are more likely to form a community. The network is then decomposed, with adjacent sub networks being loosely connected by the intergroup edges

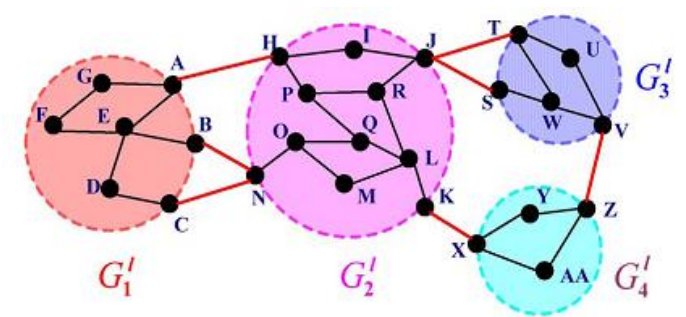

(a)

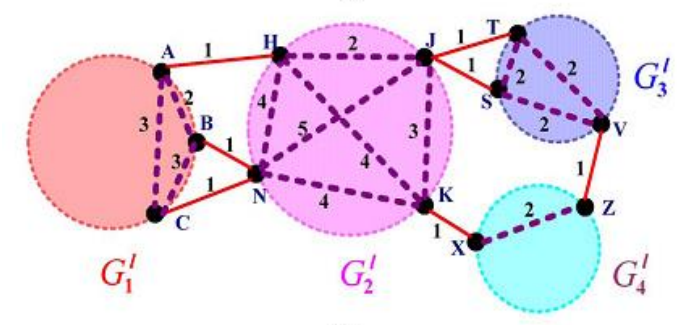

(b)

Figure 1.Two-level graph hierarchy $\left(\mathrm{G}, \mathrm{G}^{\mathrm{p}}\right)$. a) Original network b) High-level community Graph

A road network can be viewed as a graph $\mathrm{G}=(\mathrm{V}, \mathrm{E}, \mathrm{W})$, where $\mathrm{V}$ is the set of vertices representing road intersections, and $\mathrm{E}=\{\mathrm{i}, \mathrm{j} \mid(\mathrm{i}, \mathrm{j} \stackrel{\mathrm{a}}{\mathrm{V}}) \ddot{\mathrm{E}}(\mathrm{i} \neq \mathrm{j})\}$ is the set of edges representing roads. W $=\{$ wij $\mid$ wij $=f c(i, j)\}$ is the set of edge weights, where wij denotes the cost of distance.

Suppose that $\mathrm{G}(\mathrm{V}, \mathrm{E}, \mathrm{W})$ is partitioned into $\mathrm{p}$ communities at level 1 , with each community corresponding to a subgraph $G_{\mathfrak{M}}^{\mathbb{R}}\left(V_{\mathfrak{M}}^{\mathbb{l}}, E_{\mathfrak{M}}^{\mathbb{R}}, W_{\mathfrak{M}}^{\mathbb{R}}\right)$ where $1 \leq \mathrm{u} \leq \mathrm{p}$.

Definition 1: A subgraph $G_{\mathfrak{M}}^{\mathbb{R}}\left(V_{\mathfrak{M}}^{\mathbb{R}}, E_{\mathfrak{M}}^{\mathbb{R}}, W_{\mathfrak{M}}^{\mathbb{l}}\right)$ of a graph $\mathrm{G}(\mathrm{V}, \mathrm{E}, \mathrm{W})$ is a graph where $V_{\mathrm{w}}^{\mathbb{L}} \subseteq V, E_{\mathrm{w}}^{\mathbb{R}} \subseteq E$ and $W_{\mathrm{w}}^{\mathbb{L}} \subseteq W . \subseteq$

For any two nodes $\mathrm{i}, \mathrm{j}$, if $i \in V_{\mathrm{u}}^{\mathrm{l}}, j \in V_{\mathrm{u}}^{\mathbb{R}},\{i, j\rangle \in E$ then $\{i, j\rangle \in E_{\mathrm{u}}^{\mathbb{R}}$

Definition 2: Given a partition $P=\left\{G_{1}^{1}, G_{2}^{1}, G_{3}^{1}\right\}$ of $G$. We have the following:

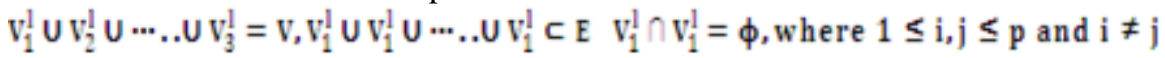


Definition 3: Given a partition $P=\left\{G_{1}^{1}, G_{2}^{1}, \ldots . G_{p}^{1}\right\}$ of $G$, a node $i$ is said to be adjacent to a node $\mathrm{j}$ if an edge exists between $\mathrm{i}$ and $\mathrm{j}$ in $\mathrm{G}$. In addition, the adjacent node set for node $\mathrm{i}$ is defined by

$A D J(i)=\{i((i, j) \Delta \in E$ or $(j, i) \in E) \wedge(i, j \in V)\}$. For node $i \in V_{u}^{l}$, if there exists a node $j \in A D J(i)$ $c_{i}^{\mathbb{R}} \neq c_{j}^{\mathbb{R}}$ Then node $I$ and named border node for. The border node set for $G_{\mathrm{w}}^{\mathbb{L}}$ is denoted by BORDER ( $\left.G_{\mathrm{W}}^{\mathbb{D}}\right)$. Two subgraphs $G_{\mathrm{W}}^{\mathbb{R}}$ and $G_{v}^{\mathbb{R}}$ are said to be adjacent if

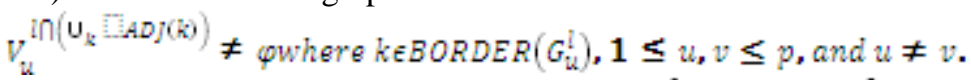

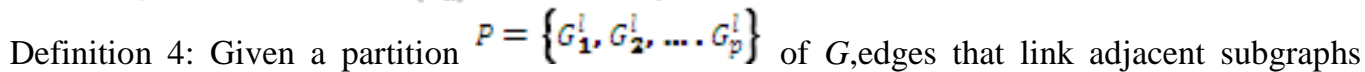
$G_{\mathrm{w}}^{\mathbb{R}}$ and $G_{\mathrm{w}}^{\mathbb{R}}$ are called the intercommunity edge set between $G_{\mathrm{w}}^{\mathbb{R}}$ and $G_{\mathbb{v}}^{\mathbb{P}}$ and the set is denoted by

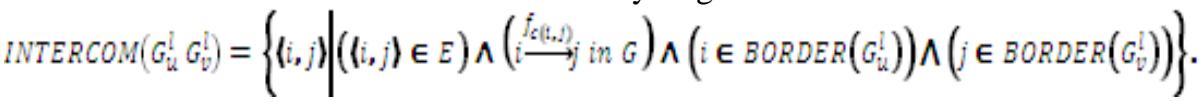

Generally, with the increase in hierarchy level, the corresponding graph contains far fewer nodes and edges. As shown in Fig.1, the graph G contains 27 nodes and 36 edges, whereas its high-level community graph GP contains only 12 nodes and 20 edges. Thus, by switching to a higher level graph hierarchy, the search space can be greatly reduced, and the time efficiency will be improved.

\section{Proposed Route Selection System}

The proposed system is executed locally for every single vehicle. It finds directions with minimum costs based on the importance rates of user desired Parameters. The architecture of the proposed system is presented in Fig 2. In this system, the traffic signal is provided by a TCC and contains current traffic data which is updated regularly. Memory of the system comprises statistical data, average speed of the vehicle, current saved traffic data, current time, and so forth,. WSN used for traffic estimation of coming minutes. This estimation is done by considering the vehicle average speed, the distance between junctions, and the current traffic flow. By taking into account the predicted traffic data and estimated journey time delay, correspondent predicted traffic data are used in the system.

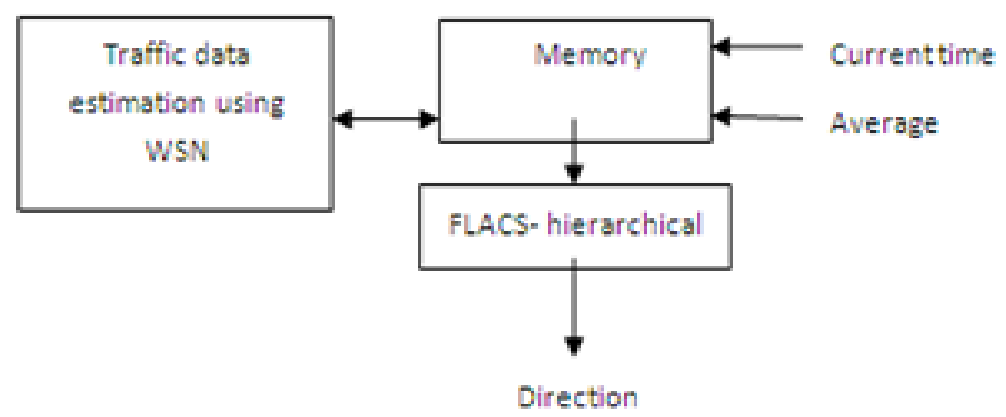

Figure 2. Proposed FLACS-Hierarchical Route Selection System

\subsection{Fuzzy Logic-Ant Colony System-Hierarchical-Based Model}

The steps involved in the Fuzzy Logic - Ant Colony System is explained as below.

Step. 1: Community Finding:

As defined in the hierarchical graph model, the road network is abstracted and partitioned into subgraphs by using a community detection algorithm. Louvain's method [15] is adopted in this paper due to its fast execution time and the high-quality hierarchical communities produced over other algorithms, which measures the density of edges inside communities, as compared with edges between communities, with high values indicating good partitions. The algorithm alternates between two phases until the modularity does not improve. Initially, each node is in its own community. Then, during phase 1 , a node $i$ is removed from its community and placed in the community of its neighbor $j$, which yields the largest increase in modularity, as long as it is positive. This process is repeated for all nodes until no positive 
gain is available, which results in the first-level partition. Then, phase 2 builds an abstraction graph where communities are replaced by supernodes, and the multiple edges connecting neighboring communities (or the same community) are replaced by one coarse edge (or self-loop) whose weight is the sum of them. The two phases are then repeated, yielding new hierarchical levels and abstraction graphs, and finally, we get the hierarchical community structure of all levels.

Step. 2: Graph hierarchal construction: After the network is decomposed, we extract the border node set of each subgraph based on Definition 3. Then, we take the border nodes of the graph, with no edges between them, and add the so-called community edges between every pair of border nodes of each subgraph, which are equivalent to the shortest paths computed within that subgraph. Finally, we link up adjacent subgraphs through intercommunity edges. Intuitively, these intercommunity edges, together with border nodes and the preprocessing-generated community edges, construct the high-level community graph, thereby forming a two-level graph hierarchy.

Step. 3: Proposed FLACS - Hierarchical algorithm used.

a. Initialize. It is consisted of initial values of the algorithm parameters such as number of ants, evaporation coefficient and average speed of the vehicle.

b. Locate Ants. Ants are located on the start point in this stage. An active ant refers to the ant, which has not arrived to the destination yet and is not blocked in a junction. Since each ant can traverse each junction once in each iteration, an ant is blocked in a junction when it has no chance of continuing its transition toward the destination and has no possible route to move backward.

c. Construct Probability. In this step, the probability of each possible direct route is calculated based on its cost function for each active ant. The probability of displacing from junction $\mathrm{i}$ to junction $\mathrm{j}$ for ant $\mathrm{k}$

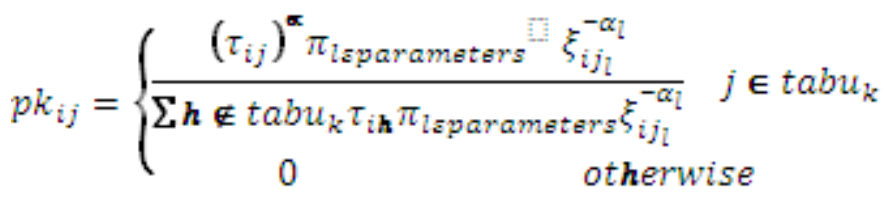

where $\hat{o} i j$ is the direct route pheromone intensity from junction $i$ to $j$. Parameter $a$ controls the importance of $\hat{o} i j$

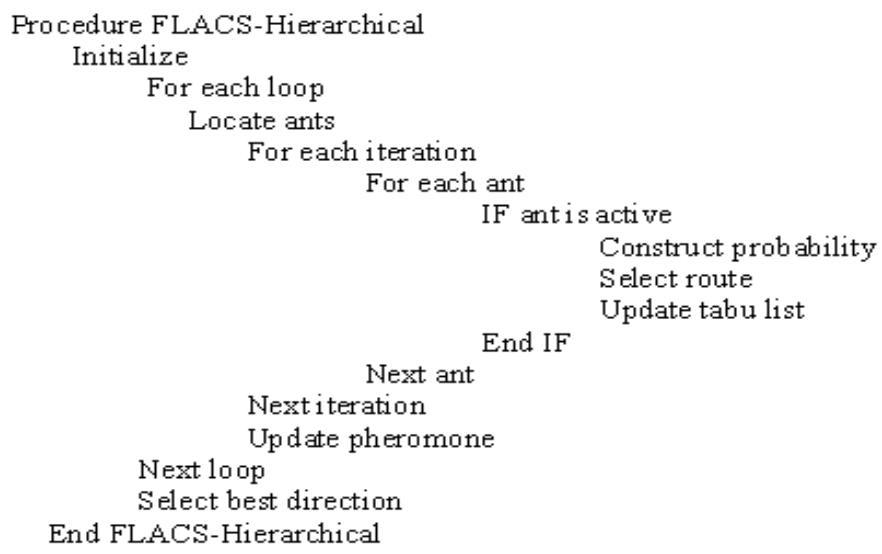

The Tabuk list is the set of direct blocked routes (visited nodes). Parameters set is a collection of most important parameters for drivers taking journeys in metropolises. For more simplicity, the "Distance," "Traffic," "Incident Risk," "Tollgate," parameters is considered in this set [16] -[18]. Cost function of each parameter $l$ is ${ }^{-\varpi_{i j_{I}}}$, where $\hat{\imath} i j l$ is normalized in $1 \leq \hat{\imath} i j l \leq 10$ and significance of each $l$ is adjustable by ál for all parameters. The considered parameters are described as follows.

(i) Distance.

$$
\hat{\imath} i \text { jdistance }=D(i, j, t)
$$

where the $D(i, j, t)$ is distance between junctions $i$ and $j$ at time $t$. Since some routes are two-way or one-way in specific hours, this parameter is a function of time. Longer distance increases total cost and therefore, decreases the probability of the longer route selection in (1). 
(ii) Traffic..

$$
\hat{\imath} i j \text { traffic }=F(i, j, t))
$$

where $F(i, j, t)$ is traffic load of the route between junctions $i$ and $j$ at time $t$. Considering this parameter in the route selection systems has many benefits such as less air pollution, less time wastage in the traffic, and less gas usage. As traffic on a route grows, total cost increases and consequently it decreases the probability of selecting that route by the system in (1).

(iii) Incident Risk.

$$
\hat{\imath} i \text { jrisk }=R(i, j, t)
$$

where $R(i, j, t)$ is risk of route between junctions $i$ and $j$ at time $t$. This parameter is a measure of incidents happening risk, which might occur on the route based on the statistical data. This parameter has direct relation with total cost, therefore risky routes have less selection probability in (1).

(iv) Tollgate.

$$
\hat{I} i \text { width }=W(i, j, t)
$$

where $\mathrm{W}(i, j, t)$ is width of route between junctions $i$ and $j$ at time $t$. This parameter is a measure number of Tollgate, which might occur on the route based on the statistical data. total cost increases and consequently it decreases the probability of selecting that route by the system in (1).

Select Route. A random parameter $0 \leq q \leq 1$ with uniform probability is compared with the parameter $Q$, where $0 \leq Q \leq 1$ and is usually fixed to 0.9[19]. The comparison result between $Q$ and $q$ picks up one of the two selection methods for the active ant to continue its route to the next junction as

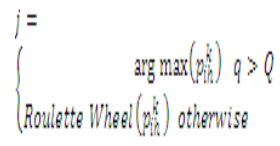

If $q$ is greater than $Q$, active ant selects the route with the highest probability, otherwise, RouletteWheel rule is selected to choose the next junction through probabilities.

Update Tabu List. In this step, the route (selected node) which ant $k$ has been chosen is added to the tabu list in order not to be selected again. Furthermore, its probability will not be calculated anymore. If ant $k$ has arrived at the destination or is blocked in a junction, it is omitted from the active ant list. In other words, this step deactivates the blocked or arrived ant in the current iteration.

Update Pheromone. The ACS pheromone system is consisted of two main rules: first is applied within the subgraph (with in community) constructing solutions (local pheromone update rule) and the other rule is applied after all ants have finished hole hierarchical community constructing a solution (global pheromone update rule). The pheromone amount of the route between junctions $i$ and $j$ is updated for ant $k$ as

$$
\begin{aligned}
& \tau_{\text {ij }}^{\text {new }}= \\
& p_{\text {ij }}+(10 \mathrm{~N} \Delta \mathrm{\tau})
\end{aligned}
$$

The FL system is illustrated in Figure 3. Inputs of the FL system are the total amount of "Distance," "Traffic," "Tollgate," and "Incident risk" of the direction which ant $k$ has selected. By considering computing complexities, only two input fuzzy sets, "Low" and "High", are defined for each input. In these functions, it is assumed that if total instance of selected direction is more than $80 \%$ of maximum total visited distance by ants in the same loop, its membership function for "High" is unity whereas when total distance of selected direction is less than $20 \%$ of maximum total visited distance by ants in the same loop, its membership function for "Low" is unity.

The similar definitions are considered for the "Traffic" and "Incident Risk" parameters. Four fuzzy sets are considered for the output variable as in Figure 4. Levels 1-4 represent different levels of pheromone density, which are "Very Weak," "Weak," "Strong," and "Very Strong," respectively. 
At the final stage, with respect to the most defuzzification techniques mentioned in the "center of gravity" method is employed in this FL system in order to resolve a single output value from the fuzzy set. Since important routes of the parameters are different and are defined by different users, various fuzzy rules are predefined in the system. Therefore, according to the preferences of each user, appropriate fuzzy rules are loaded into the FL system.. As an example,

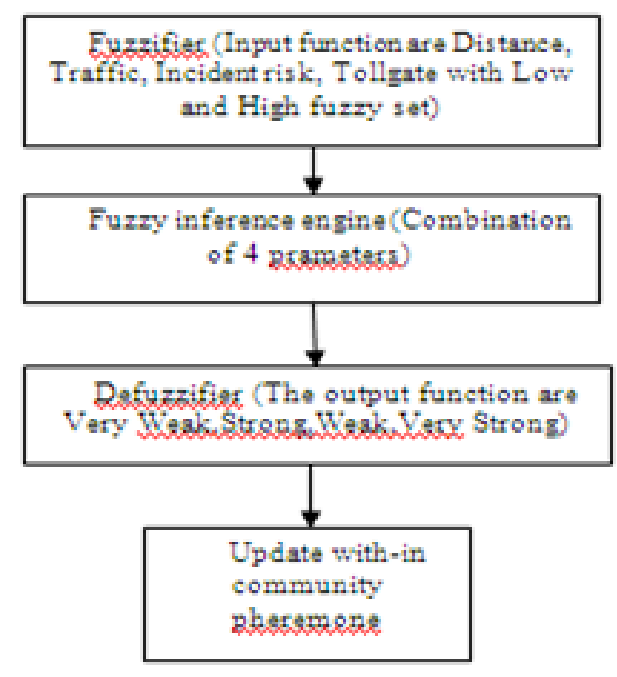

Figure 3. Architecture of FLACS Hierarchical System

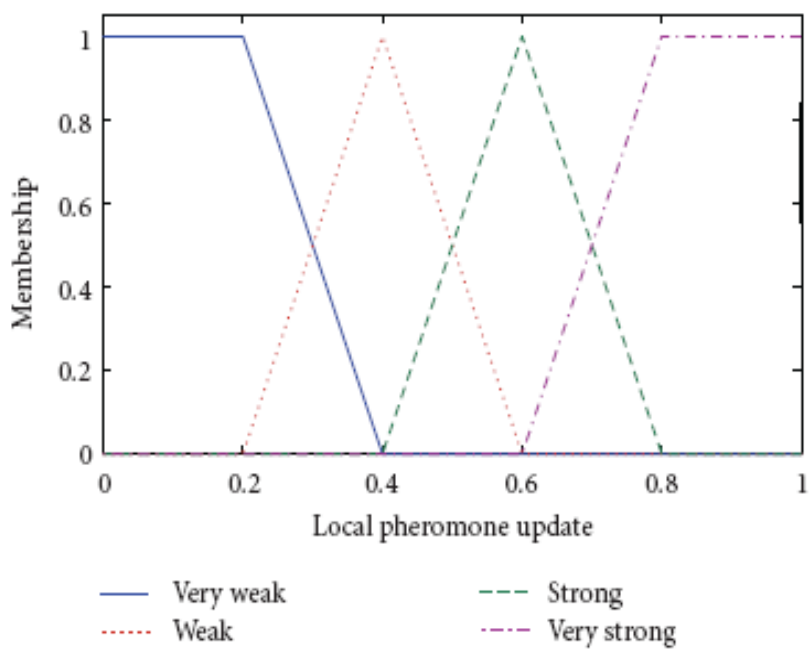

Figure 4. Membership function of within community (Local ) pheromone updating

IF-THEN rules set of a specific parameters importance rate set is presented in Table 1 . In this example, preferences of the user for the "Distance," "Traffic," "Incident Risk" and "Tollgate" parameters are "High," "Low," "Low," and "Low" respectively. Therefore, directions with closer costs to the preferences achieve more local pheromone update. This is while routes with the "Low," "High," "High," and "High" preferences are achieving the least local pheromone update. The traditional last step of each completed loop is between community (global pheromone) updating defined as

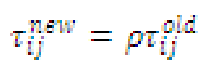

where $0<\tilde{n}<1$ is the evaporation coefficient and is usually set to 0.9 .

Select Best Direction. After $m$ loops, direction with the lowest cost from origin to destination is recommended by the system.

\section{Constructing Hierarchical Community}

First the user should identify within-community node pairs and near optimal routes for betweencommunity node pairs on large-scale road networks based on user parameter. If the source and destination are in two distinct communities, first finding the shortest path with-in community subgraph. Next finding the shortest path to the border nodes in the high-level community graph. In Figure. 5 shows 15 junctions. The Source junction is $\mathrm{S}$ and the Destination is D . start time is 4:00 PM and average speed of vehicle is considered $40 \mathrm{~km} / \mathrm{h}$ as default. 


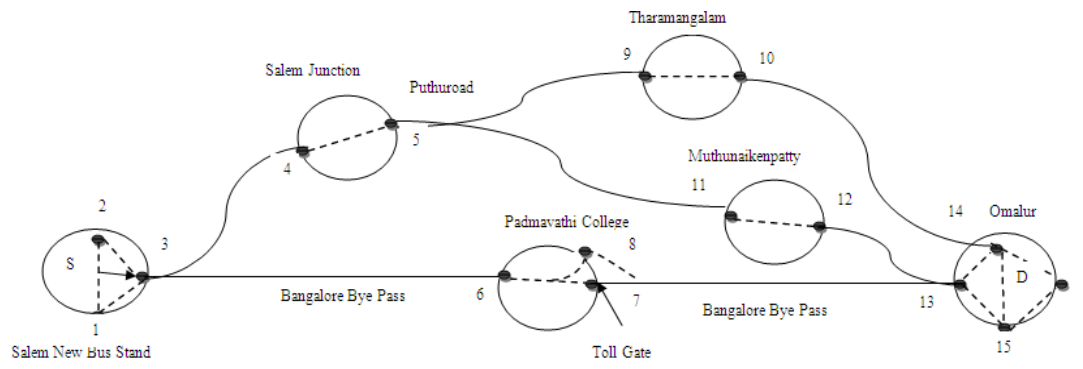

Figure 5. Using FLACS-hierarchical algorithm

Table 1. The IF-THEN rules set for FLACS system.

\begin{tabular}{|c|c|c|c|c|c|}
\hline & IF & & & & THEN \\
\hline Rule no. & Distance & Traffic & Incident Risk & Tollgate & $\begin{array}{l}\text { With-in } \\
\text { community } \\
\text { (Local) } \\
\text { Pheromone } \\
\text { Update }\end{array}$ \\
\hline 1 & High & Low & Low & Low & Very Strong \\
\hline 2 & High & High & Low & Low & Strong \\
\hline 3 & High & Low & High & Low & Strong \\
\hline 4 & Low & Low & Low & Low & Strong \\
\hline 5 & Low & Low & High & Low & Weak \\
\hline 6 & Low & High & Low & Low & Weak \\
\hline 7 & High & High & High & Low & Weak \\
\hline 8 & Low & High & High & Low & Very weak \\
\hline 9 & High & Low & Low & High & Strong \\
\hline 10 & High & High & Low & High & Weak \\
\hline 11 & High & Low & High & High & Weak \\
\hline 12 & Low & Low & Low & High & Strong \\
\hline 13 & Low & Low & High & High & Weak \\
\hline 14 & Low & High & Low & High & Weak \\
\hline 15 & High & High & High & High & Very weak \\
\hline 16 & Low & High & High & High & Very weak \\
\hline
\end{tabular}

$4 \mathrm{O} / \mathrm{D}$ pairs are randomly selected. Average of their cost averages for different number of cycles and a set of user preferences $($ Distance $=$ High; Traffic $=$ Low; Incident Risk $=$ Low Tollgate $=$ Low) is evaluated. As presented this is while the FLACS-hierarchical system has Time (sec) in the Table 2.

Table 2. The O/D pair comparison

\begin{tabular}{lllll}
\hline Route selection system & O/D Pair & Direction & Cost & Time(Sec) \\
\hline & & S $-3-4-5-11-12-13-\mathrm{D}$ & High & 2.700 \\
& & S $-2-6-8-13-\mathrm{D}$ & $\begin{array}{l}\text { With Toll gate } \\
\text { cost }\end{array}$ & 1.200 \\
FLACS-Hierarchical & S/D & S $-2-6-7-13-\mathrm{D}$ & & \\
& & S $-3-4-5-9-10-14-\mathrm{D}$ & $\begin{array}{l}\text { Without } \\
\text { Tollgate cost }\end{array}$ & 1.500 \\
& & High & \\
& & & & 5.100
\end{tabular}

Using by FLACS-hierarchical system faster with a least cost average than the other systems, compare with time wise the direction $(S-2-6-8-13-\mathrm{D})$ have to select but this one having the Toll gate, so the Dynamic direction $(S-2-6-7-13-\mathrm{D})$ selected. 


\section{Conclusion}

The proposed system in this paper introduces a dynamic route selection system which employs Fuzzy Logic (FL) and Ant Colony System (ACS) for multi parameter route selection in urban areas. This system considers a set of important parameter for city travelers: "Distance," "Traffic," "Tollgate," and "Incident Risk." In this work, costs of possible routes are calculated based on the adjusted parameters of the user. Then direction with the optimum cost is selected by using the proposed fuzzy logic-ant colony system with Hierarchical community (FLACS-Hierarchical) algorithm. For real-time applications, fuzzy logic is considered as a management mechanism for the proposed ACS local pheromone updating. This technique improves ACS performance and prepares a real applicable dynamic system for different regions. The proposed system can have lots of real-time applications for emergency services, tourist guides, and generally for anyone who wants to have a low-cost, safe, and comfortable journey.

\section{References}

[1] R.V.Helgason., J. L Kennington, and B. D Stewart ., "The one-to-one shortest-path problems: An empirical analysis with the two-tree Dijkstra algorithm," Comput. Optim. Appl., vol. 2, no. 1, pp. 47-75, Jun. 1993.

[2] L. Sun. D. Fu, and. L. R Rilett, "Heuristic shortest path algorithms for transportation applications: State of the art," Comput. Oper. Res., vol. 33, no. 11, pp. 3324-3343, Nov. 2006.

[3] B. Liu , "Route finding by using knowledge about the road network," IEEE Trans. Syst., Man, Cybern. A, Syst., Humans, vol. 27, no. 4, pp. 436-448,Jul. 1997.

[4] G. R Jagadeesh , T. Srikanthan, and K. H Quek, "Heuristic techniques for accelerating hierarchical routing on road networks," IEEE Trans. Intell.Transp. Syst., vol. 3, no. 4, pp. 301-309, Dec. 2002.

[5] Paper in the title of "Optimized Retransmission Mechanism to Prevent Wastage of Spectrum in Seamless Mobility Handover" Published in International Journal of Applied Engineering Research, ISSN 0973-4562 Vol. 10 No.5 (2015) pp. 3906-3910.P. Sanders and D. Schultes, "Highway hierarchies hasten exact shortest path queries," in Proc. ESA, Mallorca, Spain, Oct. 2005, pp. 568-579.

[6] D. Schultes and P. Sanders, "Dynamic highway-node routing," in Proc.6th Workshop Exp. Algorithms, Rome, Italy, Jun. 2007, pp. 66-79.3.

[7] Qing Song and Xiaofan Wang,"Efficient Routing on Large Road networks desired using Hierarchical communities",IEEE transaction on intelligent transaction systems,vol.12 n0.1,March. 2011

[8] A. Jayanthiladevi \& G. M. kadhar Nawaz, "An Efficient Utilization Of Spectrum In Seamless Mobility By Using Retransmission Rerouting Mechanism In Mobile IP”, Journal Of Scientific And Industrial Research, Sep-2015, NISCAIR-CSIR, India.Z. Wang ., O. Che , Chen L, and Lim A, "An efficient shortest path computation system for real road networks," in Proc. IEA/AIE, Annecy, France,Jun. 2006, pp. 711-720.

[9] Y. Huang, N. Jing ., and E. A Rundensteiner., "Effective graph clustering for path queries in digital map database," in Proc. CIKM, Rockville,MD, Nov. 1996, pp. 215-222.

[10] M. B Habbal , H. N Koutsopoulos, and S. R Lerman ., "A decomposition algorithm for the all-pairs shortest path problem on massively parallel computer architectures," Transp. Sci., vol. 28, no. 4, pp. 292-308,Nov .1994 .

[11] S. Idwan. and W. Etaiwi ., "Computing breadth first search in large graph using hMetis partitioning," Eur. J. Sci. Res., vol. 29, no. 2, pp. 215-221,Jul. 2009.

[12] S. Jung, and S. Pramanik, "An efficient path computation model for hierarchically structured topographical road maps," IEEE Trans. Knowl. DataEng., vol. 14, no. 5, pp. 1029-1046, Sep .2002.

[13] V. D. Blondel, J. L. Guillaume, R. Lambiotte, and E. Lefebvre, "Fast unfolding of communities in large networks," J. Stat. Mech., vol. 2008,no. 10, p. P10 008, Oct. 2008.

[14] H. Salehinejad., F. Pouladi, and S. Talebi, "A new route selection system: multiparameter ant algorithm based vehicle navigation approach," in Proceedings of the International Conference on Computational Intelligence for Modeling, Control and Automation, pp. 1089-1094, IEEE Computer Society,

[15] H. Salehinejad and S. Talebi, "A new ant algorithm based vehicle navigation system: a wireless networking approach," in Proceedings of the International Symposium on Telecommunications

[16] H. Salehinejad., H. Nezamabadi-Pour, S. Saryazdi., and F. Farrahi-Moghaddam., "Combined A*-ants algorithm: a new multi-parameter vehicle navigation scheme," in Proceedings of the 16th Iranian Conference on Electrical Engineering (ICEE '08), pp. 154-159, Tehran, Iran. 2008.

[17] Maniezzo.V, Gambardella.L.M, and De Luigi.F, Ant Colony Optimization, New Optimization Techniques in Engineering, Springer, Berlin, Germany. 2004.

APTIKOM J. CSIT Vol. 1, No. 1, 2016 : $35-40$ 